\title{
Effects of the functional HOTAIR rs920778 and rs12826786 genetic variants in glioma susceptibility and patient prognosis
}

\author{
Ana Xavier-Magalhães ${ }^{1,2}$ Ana I. Oliveira ${ }^{1,2}$ Joana Vieira de Castro ${ }^{1,2}$ - Marta Pojo ${ }^{1,2}$ - Céline S. Gonçalves ${ }^{1,2}$. \\ Tatiana Lourenço $^{1,2} \cdot$ Marta Viana-Pereira $^{1,2} \cdot$ Sandra Costa $^{1,2} \cdot$ Paulo Linhares $^{3} \cdot$ Rui Vaz $^{3} \cdot$ Rui Nabiço $^{4}$. \\ Júlia Amorim ${ }^{4}$ Afonso A. Pinto ${ }^{5}$ Rui M. Reis ${ }^{1,2,6} \cdot$ Bruno M. Costa ${ }^{1,2}$
}

Received: 30 June 2016 / Accepted: 21 December 2016

(C) Springer Science+Business Media New York 2017

\begin{abstract}
Abnormal expression of the long non-coding RNA HOX transcript antisense intergenic RNA (HOTAIR) is oncogenic in several human cancers, including gliomas. The HOTAIR single nucleotide polymorphisms (SNPs) rs920778 (C>T) and rs12826786 (C > T) present in the intronic enhancer and promoter regions of HOTAIR, respectively, are associated with expression, cancer susceptibility, and patient prognosis in some tumor types. However, the relevance of these HOTAIR SNPs has not been studied in glioma. Here, we report a case-control study comprising 177 Portuguese glioma patients and 199 cancer-free controls. All subjects were genotyped by PCR and
\end{abstract}

Ana Xavier-Magalhães and Ana I. Oliveira have contributed equally for this work.

Electronic supplementary material The online version of this article (doi:10.1007/s11060-016-2345-0) contains supplementary material, which is available to authorized users.

Bruno M. Costa

bfmcosta@med.uminho.pt

1 Life and Health Sciences Research Institute (ICVS), School of Medicine, University of Minho, Campus de Gualtar, 4710-057 Braga, Portugal

2 ICVS/3B's - PT Government Associate Laboratory, University of Minho, Braga/Guimarães, Campus de Gualtar, 4710-057 Braga, Portugal

3 Department of Neurosurgery, Hospital S. João, Porto, Portugal

4 Department of Oncology, Hospital de Braga, Braga, Portugal

5 Department of Neurosurgery, Hospital de Braga, Braga, Portugal

6 Molecular Oncology Research Center, Barretos Cancer Hospital, 1331 Barretos-SP - CEP 14.784-400, São Paulo, Brazil restriction fragment length polymorphism (RFLP). No statistically significant differences were found in the genotype or allele distributions of either rs920778 or rs 12826786 between glioma patients and controls, suggesting these SNPs are not associated with glioma risk. No significant associations were found between rs920778 variants and HOTAIR expression levels, while rs12826786 CT genotype was associated with increased intratumoral HOTAIR RNA levels when compared to TT genotype $(p$-value $=0.04)$. Univariate (Log-rank) and multivariate (Cox proportional) analyses showed both rs920778 CT and rs12826786 CT genotypes were significantly associated with longer overall survival of WHO grade III anaplastic oligodendroglioma patients. Our results suggest that HOTAIR SNPs rs920778 and rs12826786 do not play a significant role in glioma susceptibility, but may be important prognostic factors in anaplastic oligodendroglioma patients. Future studies are warranted to validate and expand these findings, and to further dissect the importance of these SNPs in glioma.

Keywords Glioma $\cdot$ HOTAIR $\cdot$ SNP $\cdot$ Prognosis · Rs920778 · Rs12826786

\section{Introduction}

The recent advent of new sequencing technologies and genome-wide analysis has indicated that the vast majority of the genome is transcribed into non-coding transcripts, and that only $2 \%$ of the genome accounts for protein-coding transcripts, highlighting the importance of better understanding the functions of non-protein coding genes [1, 2]. Among non-coding RNAs, those longer than 200 nucleotides-termed long non-coding RNAs (IncRNAs) - have emerged as highly attractive to cancer research due to their 
ability to local or global regulation of gene expression via epigenetic, transcriptional or post-transcriptional mechanisms [3]. Indeed, several IncRNAs were found to have crucial roles in controlling cellular pathways and gene expression programs, thus impacting the oncogenic processes by presenting either oncogenic or tumor suppressive functions [4]. The HOX Transcript Antisense Intergenic RNA (HOTAIR) is a trans-acting lncRNA able to reprogram chromatin organization throughout the genome, as it is scaffold for the binding of Polycomb Repressive Complex (PRC2) in its $5^{\prime}$ domain, while interacting on the $3^{\prime}$ domain with LSD1/REST/CoREST complex [5, 6]. The oncogenic roles of HOTAIR have been extensively described in several tumor types, and its abnormal expression was associated with patient poor prognosis, tumor growth, aggressiveness, progression, recurrence, metastatic dissemination, invasion, and chemoresistance (reviewed in [7, 8]).

Gliomas are the most frequent primary malignant tumors of the central nervous system in adults, and are divided in 2 main histological subtypes, including astrocytomas and oligodendrogliomas [9]. Astrocytomas represent the vast majority of all diagnosed gliomas (about $70 \%$ ), and are graded from I to IV according to the World Health Organization (WHO) [10]. Glioblastoma (GBM; astrocytoma WHO grade IV) is the most frequent and lethal, accounting for more than $50 \%$ of all glial tumor types [11], and is characterized by rapid growth and diffuse invasiveness of the adjacent brain parenchyma [10]. While presenting different molecular profiles resulting from distinct etiological mechanisms, no environmental factors have been firmly associated with the development of glioma, with exception of exposure to high-dose therapeutic radiation. Other putative causes may include familial aggregation, genetic syndromes, telomere maintenance and genetic polymorphisms [12-14]. The importance of inherited genetic variants in glioma risk has been highly addressed in both candidate-gene and genomewide association studies (GWAS) approaches, which revealed single nucleotide polymorphisms (SNPs) in cancer-related genes associated with glioma risk (e.g., TERT, EGF, EGFR, TGF- $\beta 1, C C D C 26, C D K N 2 A / C D K N 2 B$, PHLDB1 and RTEL1 [15-18]).

Although HOTAIR expression has been reported as crucial in the carcinogenic process of many cancer types, and its abnormal expression was associated with patient poor prognosis, tumor grade, growth and invasion, and as a putative therapeutic target in glioma [19-21], few studies have focused on the significance of SNPs in HOTAIR affecting cancer risk [22-28]. Critically, no studies have evaluated the impact of HOTAIR SNPs in glioma risk and patient prognosis. In this context, we evaluated how previously described HOTAIR SNPs rs920778 and rs12826786 may associate with glioma susceptibility and patient prognosis.

\section{Materials and methods}

\section{Study population}

This case-control study comprised 177 Portuguese glioma patients from Hospital de Braga, Hospital de São João, and Hospital de Santo António, diagnosed between 2004 and 2015, and 199 cancer-free individuals randomly selected from blood donors as controls. All participants were of Caucasian ethnic background. Tumors were classified according to WHO grading system [29], and clinicopathological data is summarized in Table 1 . The study was conducted according to institutional and European Union ethical standards, and all subjects ensured written informed consent to participate in research studies.
Table 1 Clinicopathologic features of glioma patients and cancer-free controls

\begin{tabular}{|c|c|c|c|c|c|}
\hline \multirow[t]{2}{*}{ Groups (WHO grade) } & \multirow{2}{*}{$\begin{array}{l}\text { Number } \\
\text { of cases }\end{array}$} & \multicolumn{2}{|l|}{ Age, $y$} & \multicolumn{2}{|l|}{ Gender } \\
\hline & & mean $\pm \mathrm{SD}$ & $p$-value & $\mathrm{M} / \mathrm{F}$ ratio & $p$-value \\
\hline Controls & 199 & $46.4 \pm 14.0$ & N/A & 1.88 & N/A \\
\hline Gliomas (II-IV) & 177 & $57.7 \pm 12.4$ & $p \leq 0.001$ & 1.68 & $p=0.598$ \\
\hline Astrocytoma (II-IV) & 137 & $58.3 \pm 12.1$ & $p \leq 0.001$ & 1.85 & $p=0.945$ \\
\hline Diffuse astrocytoma (II) & 7 & $53.1 \pm 13.3$ & $p=0.153$ & 1.33 & $p=0.656$ \\
\hline Anaplastic astrocytoma (III) & 5 & $56.0 \pm 9.9$ & $p=0.080$ & 0.25 & $p=0.037$ \\
\hline Glioblastoma (IV) & 118 & $59.0 \pm 12.1$ & $p \leq 0.001$ & 2.11 & $p=0.654$ \\
\hline Gliosarcoma (IV) & 7 & $53.7 \pm 12.5$ & $p=0.131$ & 1.33 & $p=0.656$ \\
\hline Oligodendroglioma (II-III) & 38 & $55.9 \pm 13.5$ & $p \leq 0.001$ & 1.24 & $p=0.238$ \\
\hline Oligodendroglioma (II) & 8 & $46.5 \pm 14.3$ & $p=0.680$ & 0.33 & $p=0.020$ \\
\hline Anaplastic oligodendroglioma (III) & 30 & $58.5 \pm 12.3$ & $p \leq 0.001$ & 1.73 & $p=0.831$ \\
\hline Anaplastic oligoastrocytoma (III) & 2 & $49.5 \pm 19.1$ & $p=0.696$ & 1.00 & $p=0.652$ \\
\hline
\end{tabular}

$F$ female, $M$ male, N/A not Applicable, $S D$ standard deviation 


\section{SNP genotyping}

Genomic DNA was collected from peripheral blood leukocytes by either proteinase $\mathrm{K} /$ chloroform/isopropanol treatment or Citogene ${ }^{\circledR}$ DNA isolation kit (Citomed). HOTAIR SNPs rs920778 $(\mathrm{C}>\mathrm{T})$ and rs12826786 $(\mathrm{C}>\mathrm{T})$ were genotyped through PCR-based restriction fragment length polymorphism (RFLP). Fifty ng of DNA were used for PCR amplification with KAPA Taq DNA Polymerase (Kapa Biosystems). Primers sequences (following previous publications $[22,23])$ and PCR conditions are listed in Supplementary Table 1 . Ten $\mu \mathrm{L}$ of PCR product were enzymatically digested at $37^{\circ} \mathrm{C}$ for 5 or 30 min with FastDigest MspI (Thermo Scientific, rs920778) or FastDigest BglII (Thermo Scientific, rs12826786), respectively. Digestion products were resolved in $4 \%$ agarose gel stained with GreenSafe Premium (Nzytech). For quality control, 20 cases were confirmed by Sanger Sequencing, and the reproducibility was $100 \%$.

\section{Intratumoral HOTAIR expression levels}

Total RNA was collected from the available glioma tissues from patients from Hospital de Braga using TRIzol method (Invitrogen), and cDNA was synthesized using High Capacity cDNA Reverse Transcription kit (Applied Biosystems) according to the manufacturer's protocol. Gene expression levels were assessed by quantitative realtime PCR (qPCR; Kapa Biosystems) with the following set of primers: Sense- CAGTGGGGAACTCTGACTCG and Antisense- GTGCCTGGTGCTCTCTTACC, and normalized to the expression of HPRT1 (Sense- TGAGGATTT GGAAAGGGTGT; Antisense- GAGCACACAGAGGGC TACAA).

\section{Statistical analysis}

Statistical analyses were performed using SPSS 21.0 (IBM SPSS Statistics, IBM ${ }^{\circledR}$ ). The different allele and genotype frequencies in glioma patients and cancer-free controls were compared by Chi square test, and nonparametric Wilcoxon-Mann Whitney test was used to compare age and sex distributions between these groups. Hardy-Weinberg equilibrium was evaluated by Chi square test, comparing the expected and observed genotype frequencies of HOTAIR SNPs in control group. Linkage disequilibrium (LD) was calculated with the SHEsis program [30]. The intratumoral expression levels of HOTAIR were compared between genotypes using Mann-Whitney test. Odds ratio (OR) and 95\% confidence intervals (CI) for assessing differences in risk and survival were calculated by univariate and a Cox multivariate logistic regression (adjusted for patient age and sex). Effect sizes were measured using Cohen's $d$ values, and large effect sizes were considered for $d \geq 0.8$ [31]. No multiple comparisons corrections were performed. Patients' overall survival was assessed by Kaplan-Meier survival curves, and the log-rank test was used to evaluate the differences. Statistical significance was considered for $p$-values $<0.05$.

\section{Results}

\section{Subject characteristics}

A total of 376 Portuguese subjects (177 glioma patients and 199 cancer-free control individuals) were genotyped to determine possible associations between HOTAIR rs920778 and rs12826786 polymorphisms and glioma risk or prognosis. The clinicopathological features of the controls and cases are shown in Table 1. Statistical analysis of age (controls: $27-85$ years; gliomas: $20-83$ years) and sex distribution between control and all glioma cases showed significant differences for age ( $p$-value $\leq 0.001)$, but not for $\operatorname{sex}(p$-value $=0.598$; Table 1$)$.

\section{Allelic frequencies and genotype distributions of HOTAIR SNPS}

The genotype and allele frequencies of HOTAIR rs920778 and rs12826786 polymorphisms in controls and glioma cases are shown in Table 2. The frequencies of TT, CT, and CC genotypes of rs 920778 were $45.2,42.2$, and $12.6 \%$ in cancer-free controls, and 46.3, 40.1, and $13.6 \%$ in glioma patients, respectively. The allelic frequencies of $\mathrm{T}$ allele were $66.3 \%$ for controls and $66.4 \%$ for glioma. Regarding rs12826786 polymorphism, the frequencies of CC, CT, and TT genotypes were $47.2,42.2$, and $10.6 \%$ in controls, and $47.5,43.5$, and $9.0 \%$ in glioma cases, respectively, being the $\mathrm{C}$ allele the most representative in the control group (68.3\%) and in glioma patients (69.2\%). The distribution of rs920778 and rs12826786 allele frequencies in the control group was in Hardy-Weinberg equilibrium ( $p$-value $=0.750$ and $p$-value $=0.935$, respectively $)$. Interestingly, these two polymorphisms were in strong linkage disequilibrium ( $D^{\prime}=0.94$ and $r^{2}=0.79$ ) in our datasets.

\section{Association between HOTAIR SNPs and glioma risk}

To define whether there was significantly increased glioma susceptibility due to the presence of particular HOTAIR rs920778 and rs12826786 genotypes, univariate (data not shown) and multivariate (Table 2) analyses were performed in all glioma and GBM patients, the most frequent subtype in our series $(n=118)$. No significant associations between these polymorphic variants and the risk for developing 
Table 2 Multivariate logistic regression analysis of the association between rs 920778 and rs12826786 polymorphisms and glioma susceptibility by WHO grades

\begin{tabular}{|c|c|c|c|c|c|}
\hline Polymorphism & Control & $\begin{array}{l}\text { Glioma (WHO } \\
\text { grade II-IV) }\end{array}$ & $\mathrm{OR}(95 \% \mathrm{CI})^{\mathrm{a}}$ & $\begin{array}{l}\text { Glioblastoma } \\
\text { (WHO grade } \\
\text { IV) }\end{array}$ & OR $(95 \% \mathrm{CI})^{\mathrm{a}}$ \\
\hline \multicolumn{6}{|l|}{ rs920778 } \\
\hline \multicolumn{6}{|l|}{ Genotypes } \\
\hline TT & 90 & 82 & - & 54 & - \\
\hline CT & 84 & 71 & $0.969(0.602-1.561)$ & 45 & $0.927(0.537-1.601)$ \\
\hline $\mathrm{CC}$ & 25 & 24 & $1.060(0.528-2.127)$ & 14 & $0.867(0.384-1.961)$ \\
\hline $\mathrm{CC}+\mathrm{CT}$ & 109 & 95 & $0.990(0.635-1.545)$ & 59 & $0.913(0.547-1.522)$ \\
\hline \multicolumn{6}{|l|}{ Alleles } \\
\hline $\mathrm{T}$ & 264 & 235 & - & 153 & - \\
\hline $\mathrm{C}$ & 134 & 119 & $1.013(0.727-1.412)$ & 73 & $0.925(0.630-1.359)$ \\
\hline Age & & & $1.063(1.045-1.081)$ & & $1.071(1.050-1.092)$ \\
\hline \multicolumn{6}{|l|}{ Sex } \\
\hline Male & 130 & 111 & - & 76 & - \\
\hline Female & 69 & 66 & $1.005(0.632-1.598)$ & 37 & $0.716(0.414-1.239)$ \\
\hline \multicolumn{6}{|l|}{ rs 12826786} \\
\hline \multicolumn{6}{|l|}{ Genotypes } \\
\hline $\mathrm{CC}$ & 94 & 84 & - & 57 & - \\
\hline $\mathrm{CT}$ & 84 & 77 & $1.040(0.651-1.661)$ & 48 & $0.966(0.565-1.654)$ \\
\hline $\mathrm{TT}$ & 21 & 16 & $0.837(0.384-1.825)$ & 8 & $0.533(0.202-1.411)$ \\
\hline $\mathrm{TT}+\mathrm{CT}$ & 105 & 93 & $0.998(0.640-1.556)$ & 56 & $0.872(0.523-1.454)$ \\
\hline \multicolumn{6}{|l|}{ Alleles } \\
\hline $\mathrm{C}$ & 272 & 245 & - & 162 & - \\
\hline $\mathrm{T}$ & 126 & 109 & $0.959(0.683-1.346)$ & 64 & $0.821(0.552-1.221)$ \\
\hline Age & - & - & $1.063(1.045-1.081)$ & - & $1.072(1.051-1.093)$ \\
\hline \multicolumn{6}{|l|}{ Sex } \\
\hline Male & 130 & 111 & - & 76 & - \\
\hline Female & 69 & 66 & $1.000(0.628-1.591)$ & 37 & $0.701(0.403-1.218)$ \\
\hline
\end{tabular}

Boldfaced values indicate statistical significant differences at $5 \%$ level

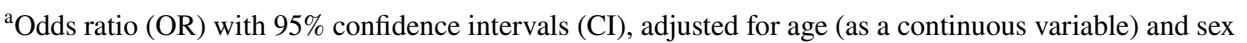

glioma or GBM were found ( $p$-value $>0.05$ ). As expected, in the multivariate analyses, increased age was associated with increased risk for developing glioma (OR 1.063; 95\% CI 1.045-1.081) or GBM (OR 1.071; 95\% CI 1.050-1.092; Table 2).

\section{Association between HOTAIR SNPs and gene expression levels in glioma specimens}

The expression of HOTAIR was evaluated in 34 glioma tumor tissues (freshly collected adequately for RNA analyses) to understand whether the rs 920778 and rs12826786 SNPs may be associated with HOTAIR mRNA levels (Fig. 1). For rs920778, there were no statistically significant differences between CC, TT and CT genotypes and HOTAIR expression (Fig. 1a). In contrast, glioma subjects with the rs 12826786 CT genotype had significantly higher tumor HOTAIR RNA levels than those with the TT genotype (Fig. 1b, $p$-value $=0.04$ ).

\section{Prognostic value of HOTAIR SNPs in glioma patients}

We next investigated the association between these HOTAIR polymorphisms and patients' overall survival in WHO grade IV GBM $(n=69)$ and WHO grade III anaplastic oligodendroglioma tumors $(n=18)$ with available follow-up data.

No statistically significant associations were found between rs920778 or rs12826786 polymorphisms and survival of GBM patients both by univariate (log rank test $p$-value $=0.458$ and $p$-value $=0.306$, respectively) and multivariate analyses adjusted for patients' age and sex (Cox regression model $p$-values $>0.05$; data not shown).

In contrast, univariate analyses for both SNPs in anaplastic oligodendroglioma patients showed that carriers of CT genotypes present longer overall survival than CC patients ( $p$-value $=0.029$ and $p$-value $\leq 0.001$, for rs920778 and rs12826786, respectively; Fig. 2) or when compared to all other patients (CT vs $\mathrm{CC}+\mathrm{TT}$; 


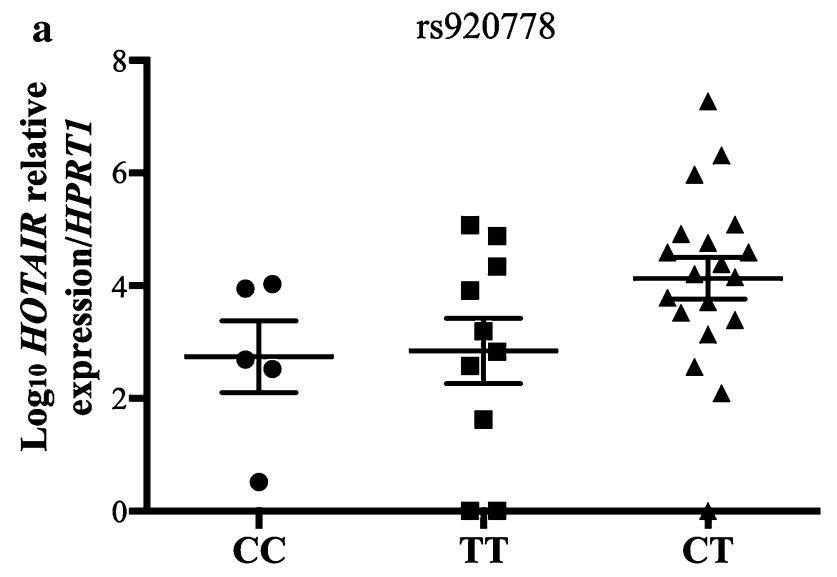

Fig. 1 HOTAIR expression levels in glioma tissues grouped by rs920778 and rs12826786 genotypes. Within rs920778 genotypes (a), HOTAIR expression levels are not statistically different (CC $\mathrm{n}=5$, TT $\mathrm{n}=10$, CT $\mathrm{n}=19$ ), whereas in rs12826786 polymorphism (b), patients presenting CT $(\mathrm{n}=19)$ genotype have increased levels of

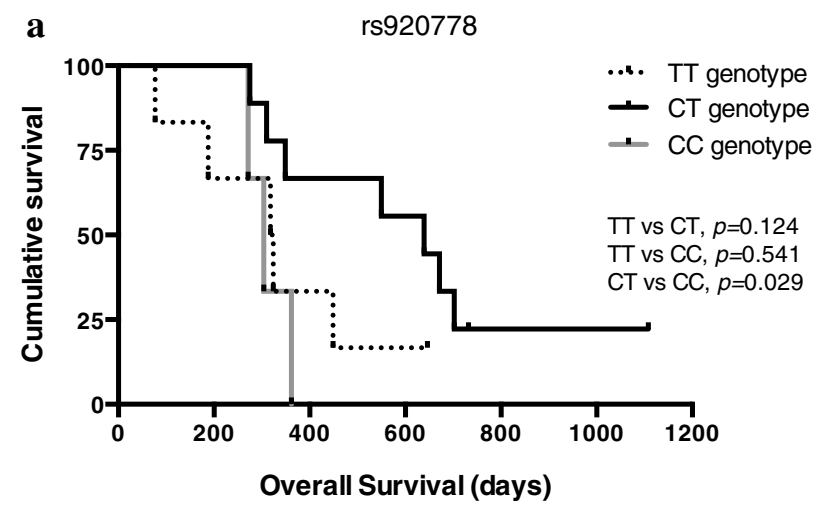

Fig. 2 Effect of HOTAIR rs920778 and rs12826786 polymorphisms in the survival of WHO grade III anaplastic oligodendroglioma patients. Kaplan-Meier survival curves indicate that a patients with CT genotype $(n=9)$ in rs920778 locus of HOTAIR present statistically significant longer overall survival than patients carrying the CC genotype $(n=3$; Log-rank test, $p$-value $=0.029)$, but not the TT genotype $(n=6$; Log-rank test, $p$-value $=0.124)$. No statistically significant associations were found between the TT and the

$p$-value $=0.043$ and $p$-value $\leq 0.001$, for rs920778 and rs12826786, respectively; data not shown). Additionally for rs12826786, CT genotype patients also presented longer overall survival when compared to TT patients ( $p$-value $=0.027$; Fig. 2b).

Accordingly, Cox multivariate analysis showed that, for both SNPs, patients with the CT genotype present statistically significant longer overall survival than CC patients, independently of age and sex ( $p$-value $=0.032$ and $p$-value $=0.010$, for rs920778 and rs12826786, respectively; Table 3). Moreover, for rs12826786, the CT genotype also conferred longer overall survival than TT

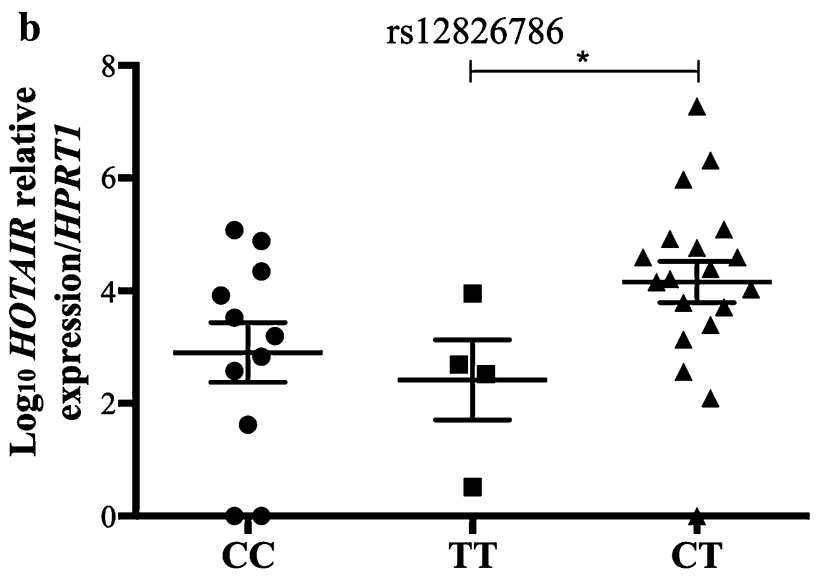

HOTAIR in comparison to TT patients $(\mathrm{n}=4)$, but not to CC patients $(\mathrm{n}=11)$. The expression levels were normalized to HPRT1 expression using the $2^{-\Delta \mathrm{C}_{\mathrm{t}}}$ method, transformed in logarithmic $\left(\log _{10}\right)$ scale, and are represented by mean \pm SEM. $* p$-value $=0.04$

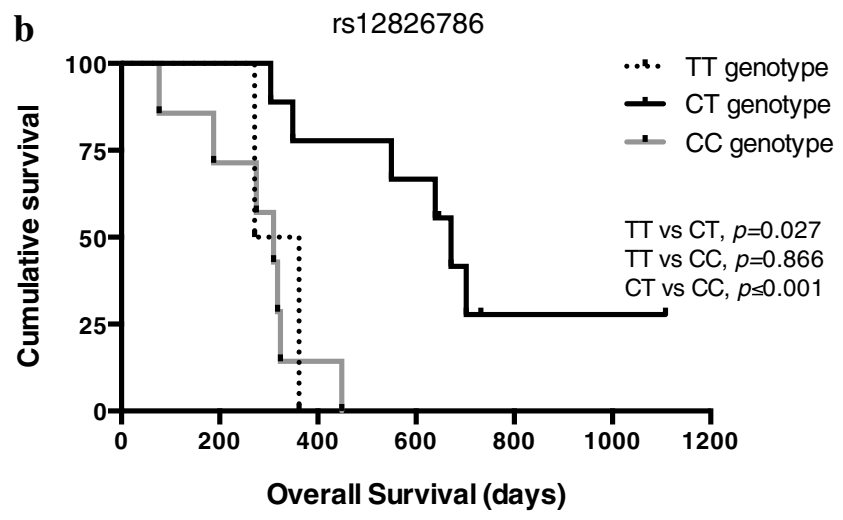

CC genotypes $(p$-value $=0.541)$. b Patients harboring the CT genotype $(n=9)$ in rs12826786 SNP have statistically significant longer overall survival than patients with the CC genotype $(n=7$; Log-rank test, $p$-value $\leq 0.001)$ and the TT genotype $(n=2$; Log-rank test, $p$-value $=0.032$ ). No statistically significant associations were found between the TT and the CC genotypes $(p$-value $=0.866)$. Tick marks indicate censored data

genotype patients ( $p$-value $=0.032$; Table 3 ) or when compared to all other genotypes $(p$-value $=0.006$; Table 3 ).

\section{Discussion}

The discovery of genetic factors associated with glioma susceptibility is required for a deeper understanding of this devastating disease, and to develop novel therapeutic approaches. HOTAIR is a known oncogenic lncRNA in several tumor types (reviewed in $[7,8]$ ) including gliomas [15-17]. The roles of HOTAIR have been widely reported 
Table 3 Multivariate Cox regression analysis of the association between rs920778 and rs12826786 polymorphisms and survival in WHO grade III anaplastic oligodendroglioma patients

\begin{tabular}{lclll}
\hline Polymorphism & $\begin{array}{c}\text { Number } \\
\text { of case }\end{array}$ & $p$-value & HR $(95 \% \mathrm{CI})^{\mathrm{b}}$ & Cohen's $d$ \\
\hline rs920778 & & & & \\
Genotypes & & & & - \\
CT & 9 & 0.094 & - & \\
CC & 3 & $\mathbf{0 . 0 3 2}$ & $\mathbf{6 . 2 9 2}(\mathbf{1 . 1 7 5 - 3 3 . 6 9 )})$ & $1.014^{\mathrm{a}}$ \\
TT & 6 & 0.267 & $2.320(0.526-10.24)$ & 0.464 \\
CC+TT & 9 & 0.070 & $3.264(0.909-11.72)$ & 0.652 \\
Age & & 0.187 & $1.046(0.978-1.119)$ & 0.025 \\
Sex & & & & \\
Male & 13 & - & - & - \\
Female & 5 & 0.236 & $2.305(0.578-9.188)$ & 0.460 \\
rs12826786 & & & & \\
Genotypes & & & & - \\
CT & 9 & $\mathbf{0 . 0 2 2}$ & - & $1.386^{\mathrm{a}}$ \\
CC & 7 & $\mathbf{0 . 0 1 0}$ & $\mathbf{1 2 . 3 4}(\mathbf{1 . 8 3 7 - 8 2 . 9 3 )}$ & \\
TT & 2 & $\mathbf{0 . 0 3 2}$ & $\mathbf{9 . 1 8 8}(\mathbf{1 . 2 0 9 - 6 9 . 8 1})$ & $1.223^{\mathrm{a}}$ \\
CC+TT & 9 & $\mathbf{0 . 0 0 6}$ & $\mathbf{1 0 . 7 9}(\mathbf{1 . 9 9 8 - 5 8 . 2 4})$ & $1.311^{\mathrm{a}}$ \\
Age & & 0.392 & $1.026(0.968-1.087)$ & 0.014 \\
Sex & & & & - \\
Male & 13 & - & - & \\
Female & 5 & 0.754 & $0.791(0.182-3.437)$ & -0.129 \\
\hline Bex & & & & \\
\hline
\end{tabular}

Bold-faced values indicate statistical significant differences at 5\% level

aValues that exceed Cohen's $d$ convention for a large effect size $(d \geq 0.8)$

${ }^{\mathrm{b}}$ Hazard ratio (HR) with $95 \%$ confidence intervals (CI), adjusted for age (as a continuous variable) and sex

in cancer, but epidemiological studies evaluating tumor susceptibility conferred by genetic polymorphisms in its locus have not been extensively assessed [18-22]. Indeed, to the best of our knowledge, the current study is the first to evaluate associations between HOTAIR SNPs (rs920778 and rs12826786) and glioma susceptibility and prognosis. We have analyzed a Portuguese population consisting of 177 glioma patients and 199 cancer-free controls. Our results showed that the allelic frequencies of HOTAIR SNPs rs920778 and rs12826786 were not significantly different between glioma patients and cancer-free controls. Importantly, for both polymorphisms, the genotypes frequencies in cancer-free controls are in agreement with those reported by the HapMap Project for subjects of European ancestry (HapMap-CEU, data not shown; http://hapmap.ncbi.nlm. nih.gov/). Considering risk assessments, our results showed no significant associations between HOTAIR rs920778 or rs12826786 and susceptibility for any WHO grade glioma. Previous studies on the contribution of rs920778 to gastric cancer in a Turkish population [25] also showed no differences between patients and cancer-free controls; however, studies in other tumor types testing the importance of rs920778 or rs12826786 in the Chinese population have shown different allele frequencies between patients and cancer-free controls [22-24]. It was also showed previously that rs920778 variants do not influence gastric cancer susceptibility [25], whereas others have showed statistically significant associations between these SNPs and risk to develop gastric cardia adenocarcinoma [22], esophageal squamous cell carcinoma (ESCC) [23], breast cancer [26-28] or gastric cancer [24]. Such discrepancies might be attributed to the different genetic backgrounds of the studied populations, different sample sizes, and/or to the variety of tumor types analyzed. Indeed, the expression patterns, the regulatory mechanisms, and the functions of lncRNAs are highly tissue-specific [32]. Because SNPs differentially influence the regulation of IncRNAs [33], in particular the SNPs in this study [22-24], it is possible that these SNPs may present distinct relevance in the context of different tumor types.

Since rs920778 and rs12826786 HOTAIR SNPs have been previously reported to be located in an intronic enhancer or in the promoter, respectively, we sought to understand if the different genotypes influence the expression levels of HOTAIR in glioma tissues. Our results showed that patients with the rs12826786 CT genotype presented increased intratumoral levels of HOTAIR, compared to TT genotype but not CC, whereas in rs920778 no differences were found. Previous reports showed increased levels of HOTAIR in TT homozygous patients with gastric cancer or gastric cardia adenocarcinoma for rs920778 [24] or rs12826786 [22], respectively, again highlighting tumor type-specific roles of SNPs.

Given that HOTAIR expression is a known negative prognostic factor in glioma [19], we evaluated the importance of rs920778 or rs12826786 genotypes in the overall survival of the glioma patients from our dataset. We did not find any associations between rs920778 and rs12826786 and overall survival for GBM patients (data not shown). Conversely, anaplastic oligodendroglioma patients presenting the CT genotype for rs 920778 or rs 12826786 had significantly longer overall survivals, both in univariate and ageand sex-adjusted multivariate analyses (Fig. 2; Table 3). Interestingly, in our GBM dataset, the TT genotype is the least frequent $(n=5 / 69)$, and these patients presented a tendency for shorter overall survival in comparison to either $\mathrm{CC}$ or CT patients. Concordantly, a report in gastric cardia adenocarcinoma [22] has shown for the rs12826786 SNP that TT genotype is the least frequent and that these patients also present a tendency to shorter overall survival in comparison to $\mathrm{CT}$ or $\mathrm{CC}$, while presenting no overall survival differences for $\mathrm{CC}$ or $\mathrm{CT}$ patients. It is important to highlight that our survival analyses are based on a small 
cohort of patients ( $\mathrm{n}=18$ for WHO grade III anaplastic oligodendroglioma and $n=69$ for WHO grade IV GBM), for which we had available follow-up information. Nonetheless, our findings that rs920778 and rs12826786 CT genotypes are associated with longer survival of WHO grade III anaplastic oligodendroglioma patients are integrally supported by complementary statistical approaches, including Log-rank tests (which take into consideration sample size), multivariate Cox regression models adjusted for potential confounding effects (like patient age and sex), as well as calculations of Hazard Ratios and Cohen's $d$ values (which relate to the effect size, and are independent of sample size; Fig. 2; Table 3). These findings suggest putative roles of these HOTAIR SNPs in the survival of anaplastic oligodendroglioma patients, warranting further validation by independent studies with larger datasets.

In conclusion, our findings add to the body of knowledge surrounding genetic polymorphisms as putative players affecting glioma risk or prognosis. We found that HOTAIR rs920778 and rs12826786 do not seem to play any major role in genetic susceptibility of glioma in the Portuguese population. In contrast, HOTAIR rs12826786 presents genotype specific effect on tumoral HOTAIR expression levels, and both SNPs showed prognostic significance in the Portuguese anaplastic oligodendroglioma population. Therefore, HOTAIR rs920778 and rs12826786 may be useful biomarkers to predict the outcome of WHO grade III anaplastic oligodendroglioma patients. Of note, these two SNPs were in strong linkage disequilibrium in the glioma population, which could partly explain the similar results obtained for both SNPs relating to risk and prognostic analysis. Further studies are required to validate and expand our results to populations with different genetic backgrounds to better understand the broader importance of these SNPs in gliomas. Taking advantage of genotyping projects (e.g., HapMap and the 1000genomes.org), it may be interesting to identify and study additional HOTAIR polymorphisms, which may have functional consequences and play a role in glioma pathophysiology.

Acknowledgements The authors would like to acknowledge the funding agencies that supported this work: Fundação para a Ciência e Tecnologia (PTDC/SAU-GMG/113795/2009 and IF/00601/2012 to B.M.C.; SFRH/BD/88220/2012 to A.X.M.; SFRH/ BD/52287/2013 to A.I.O.; SFRH/BD/88121/2012 to J.V.C.; SFRH/ BD/81042/2011 to M.P.; SFRH/BD/92786/2013 to C.S.G.; SFRH/ BD/51996/2012 to T.L.; SFRH/BPD/104290/2014 to M.V.P.; PTDC/ SAU-ONC/115513/2009 to R.M.R., Fundação Calouste Gulbenkian (B.M.C.), Liga Portuguesa Contra o Cancro (B.M.C.) and Inter-University Doctoral Programme in Ageing and Chronic Disease (PhDOC; to A.X.M. and A.I.O.). Project co-financed by Programa Operacional Regional do Norte (ON.2-O Novo Norte), Quadro de Referência Estratégico Nacional (QREN), Fundo Europeu de Desenvolvimento Regional (FEDER). The authors would like to extend their appreciation to all the patients and control subjects that kindly provided the biological samples for this study.

\section{Compliance with ethical standards}

Conflict of interest The authors disclose no conflicts of interest.

Ethical approval All procedures performed in studies involving human participants were in accordance with the ethical standards of the institutional and/or national research committee and with the 1964 Helsinki declaration and its later amendments or comparable ethical standards.

Human and animal participants This article does not contain any studies with animals performed by any of the authors.

\section{References}

1. Consortium EP (2012) An integrated encyclopedia of DNA elements in the human genome. Nature 489(7414):57-74. doi:10.1038/nature11247

2. Consortium EP, Birney E, Stamatoyannopoulos JA, Dutta A, Guigo R, Gingeras TR, Margulies EH, Weng Z, Snyder M, Dermitzakis ET et al (2007) Identification and analysis of functional elements in $1 \%$ of the human genome by the ENCODE pilot project. Nature 447(7146):799-816. doi:10.1038/nature05874

3. Mercer TR, Dinger ME, Mattick JS (2009) Long non-coding RNAs: insights into functions. Nature reviews Genetics 10(3):155-159. doi:10.1038/nrg2521

4. Huarte M, Guttman M, Feldser D, Garber M, Koziol MJ, Kenzelmann-Broz D, Khalil AM, Zuk O, Amit I, Rabani M et al (2010) A large intergenic noncoding RNA induced by p53 mediates global gene repression in the p53 response. Cell 142(3):409-419. doi:10.1016/j.cell.2010.06.040

5. Rinn JL, Kertesz M, Wang JK, Squazzo SL, Xu X, Brugmann SA, Goodnough LH, Helms JA, Farnham PJ, Segal E et al (2007) Functional demarcation of active and silent chromatin domains in human HOX loci by noncoding RNAs. Cell 129(7):13111323. doi:10.1016/j.cell.2007.05.022

6. Tsai MC, Manor O, Wan Y, Mosammaparast N, Wang JK, Lan F, Shi Y, Segal E, Chang HY (2010) Long noncoding RNA as modular scaffold of histone modification complexes. Science 329(5992):689-693. doi:10.1126/science.1192002

7. Hajjari M, Salavaty A (2015) HOTAIR: an oncogenic long noncoding RNA in different cancers. Cancer Biol Med 12(1):1-9. doi:10.7497/j.issn.2095-3941.2015.0006

8. Wu Y, Zhang L, Wang Y, Li H, Ren X, Wei F, Yu W, Wang X, Zhang L, Yu J et al (2014) Long noncoding RNA HOTAIR involvement in cancer. Tumour Biol 35(10):9531-9538. doi:10.1007/s13277-014-2523-7

9. Louis DN, Perry A, Reifenberger G, von Deimling A, FigarellaBranger D, Cavenee WK, Ohgaki H, Wiestler OD, Kleihues P, Ellison DW (2016) The 2016 World Health Organization Classification of Tumors of the Central Nervous System: a summary. Acta Neuropathol (Berl) 131(6):803-820. doi:10.1007/ s00401-016-1545-1

10. Kleihues P, Sobin LH (2000) World Health Organization classification of tumors. Cancer 88(12):2887

11. Lino MM, Merlo A (2010) PI3Kinase signaling in glioblastoma. J Neurooncol. doi:10.1007/s11060-010-0442-z

12. Ohgaki H, Kleihues $P$ (2005) Epidemiology and etiology of gliomas. Acta Neuropathol (Berl) 109(1):93-108. doi:10.1007/ s00401-005-0991-y

13. Bondy ML, Scheurer ME, Malmer B, Barnholtz-Sloan JS, Davis FG, Il'yasova D, Kruchko C, McCarthy BJ, Rajaraman P, 
Schwartzbaum JA et al (2008) Brain tumor epidemiology: consensus from the brain tumor epidemiology consortium. Cancer 113(7 Suppl):1953-1968. doi:10.1002/cncr.23741

14. Walsh KM, Wiencke JK, Lachance DH, Wiemels JL, Molinaro AM, Eckel-Passow JE, Jenkins RB, Wrensch MR (2015) Telomere maintenance and the etiology of adult glioma. Neurooncol. doi:10.1093/neuonc/nov082

15. Costa BM, Viana-Pereira M, Fernandes R, Costa S, Linhares $P$, Vaz R, Pinheiro C, Lima J, Soares P, Silva A et al (2011) Impact of EGFR genetic variants on glioma risk and patient outcome. Cancer epidemiology, biomarkers \& prevention : a publication of the American Association for Cancer Research, cosponsored by the American Society of Preventive. Int Soc Cell 20(12):2610 2617. doi:10.1158/1055-9965.EPI-11-0340

16. Vieira de Castro J, Goncalves CS, Costa S, Linhares P, Vaz R, Nabico R, Amorim J, Viana-Pereira M, Reis RM, Costa BM (2015) Impact of TGF-beta1 $-509 \mathrm{C} / \mathrm{T}$ and $869 \mathrm{~T} / \mathrm{C}$ polymorphisms on glioma risk and patient prognosis. Tumour Biology 36(8):6525-6532. doi:10.1007/s13277-015-3343-0

17. Costa BM, Ferreira P, Costa S, Canedo P, Oliveira P, Silva A, Pardal F, Suriano G, Machado JC, Lopes JM et al (2007) Association between functional EGF+ 61 polymorphism and glioma risk. Clin Cancer Res 13(9):2621-2626. doi:10.1158/1078-0432. CCR-06-2606

18. Ostrom QT, Bauchet L, Davis FG, Deltour I, Fisher JL, Langer CE, Pekmezci M, Schwartzbaum JA, Turner MC, Walsh KM et al (2014) The epidemiology of glioma in adults: a "state of the science" review. Neuro-oncol 16(7):896-913. doi:10.1093/ neuonc/nou087

19. Zhang JX, Han L, Bao ZS, Wang YY, Chen LY, Yan W, Yu SZ, Pu PY, Liu N, You YP et al (2013) HOTAIR, a cell cycle-associated long noncoding RNA and a strong predictor of survival, is preferentially expressed in classical and mesenchymal glioma. Neuro-oncol 15(12):1595-1603. doi:10.1093/neuonc/not131

20. Zhang K, Sun X, Zhou X, Han L, Chen L, Shi Z, Zhang A, Ye M, Wang Q, Liu C et al (2015) Long non-coding RNA HOTAIR promotes glioblastoma cell cycle progression in an EZH2 dependent manner. Oncotarget 6(1):537-546

21. Zhou X, Ren Y, Zhang J, Zhang C, Zhang K, Han L, Kong L, Wei J, Chen L, Yang J et al (2015) HOTAIR is a therapeutic target in glioblastoma. Oncotarget 6(10):8353-8365

22. Guo W, Dong Z, Bai Y, Guo Y, Shen S, Kuang G, Xu J (2015) Associations between polymorphisms of HOTAIR and risk of gastric cardia adenocarcinoma in a population of north China. Tumour Biol 36(4):2845-2854. doi:10.1007/s13277-014-2912-y

23. Zhang X, Zhou L, Fu G, Sun F, Shi J, Wei J, Lu C, Zhou C, Yuan Q, Yang M (2014) The identification of an ESCC susceptibility
SNP rs920778 that regulates the expression of lncRNA HOTAIR via a novel intronic enhancer. Carcinogenesis 35(9):2062-2067. doi:10.1093/carcin/bgu103

24. Pan W, Liu L, Wei J, Ge Y, Zhang J, Chen H, Zhou L, Yuan Q, Zhou C, Yang M (2015) A functional lncRNA HOTAIR genetic variant contributes to gastric cancer susceptibility. Mol Carcinog. doi:10.1002/mc.22261

25. Bayram S, Ulger Y, Sumbul AT, Kaya BY, Rencuzogullari A, Genc A, Sevgiler Y, Bozkurt O, Rencuzogullari E (2015) A functional HOTAIR rs920778 polymorphism does not contributes to gastric cancer in a Turkish population: a case-control study. Fam Cancer. doi:10.1007/s10689-015-9813-0

26. Bayram S, Sumbul AT, Batmaci CY, Genc A (2015) Effect of HOTAIR rs920778 polymorphism on breast cancer susceptibility and clinicopathologic features in a Turkish population. Tumour Biol 36(5):3863-3870. doi:10.1007/s13277-014-3028-0

27. Bayram S, Sumbul AT, Dadas E (2015) A functional HOTAIR rs $12826786 \mathrm{C}>\mathrm{T}$ polymorphism is associated with breast cancer susceptibility and poor clinicopathological characteristics in a Turkish population: a hospital-based case-control study. Tumour Biol. doi:10.1007/s13277-015-4430-y

28. Yan R, Cao J, Song C, Chen Y, Wu Z, Wang K, Dai L (2015) Polymorphisms in lncRNA HOTAIR and susceptibility to breast cancer in a Chinese population. Cancer Epidemiol 39(6):978985. doi:10.1016/j.canep.2015.10.025

29. Louis DN, Ohgaki H, Wiestler OD, Cavenee WK, Burger PC, Jouvet A, Scheithauer BW, Kleihues P (2007) The 2007 WHO classification of tumours of the central nervous system. Acta Neuropathol (Berl) 114(2):97-109. doi:10.1007/ s00401-007-0243-4

30. Shi YY, He L (2005) SHEsis, a powerful software platform for analyses of linkage disequilibrium, haplotype construction, and genetic association at polymorphism loci. Cell Res 15(2):97-98. doi:10.1038/sj.cr.7290272

31. Cohen J (1988) Statistical power analysis for the behavioral sciences. 2nd edn. Lawrence Erlbaum Associates, Hillsdale

32. Mercer TR, Dinger ME, Sunkin SM, Mehler MF, Mattick JS (2008) Specific expression of long noncoding RNAs in the mouse brain. Proc Natl Acad Sci USA 105(2):716-721. doi:10.1073/pnas.0706729105

33. Wu H, Zheng J, Deng J, Hu M, You Y, Li N, Li W, Lu J, Zhou Y (2013) A genetic polymorphism in lincRNA-uc003opf.1 is associated with susceptibility to esophageal squamous cell carcinoma in Chinese populations. Carcinogenesis 34(12):2908-2917. doi:10.1093/carcin/bgt252 